\title{
Test rapido per patogeni respiratori e prescrizione di antibiotici in Pronto Soccorso nel bambino con patologia respiratoria acuta
}

Suchitra Rao, Molly M. Lamb, Angela Moss, et al.

Rapid respiratory virus testing and antibiotic prescribing among children with acute respiratory illness presenting to the ED JAMA Network Open. 2021;4(6):e2111836. doi:10.1001/jamanetworkopen.2021.11836

\author{
Rubrica L'articolodelmese \\ a cura di Daniele De Brasi
}

\begin{abstract}
A fronte di un elevata prescrizione di antibiotici in Pronto Soccorso (PS) per $i$ bambini con malattia respiratoria acuta, alcuni studi riportano viceversa una riduzione del loro utilizzo nei pazienti ricoverati dopo il test rapido per patogeni respiratori (RRP). Obiettivo dello studio è stato quello di determinare se l'utilizzo degli RRP porti ad un decremento della prescrizione di antibiotici nei bambini con malattia simil-influenzale (ILI) visitati in PS. Sono stati reclutati pazienti 1-18 anni con accesso al PS nel periodo dicembre 2018 - marzo 2019 in uno studio randomizzato caso-controllo. I pazienti sono stati randomizzati in due gruppi, un gruppo di intervento ed uno di controllo (risultato test non noto). E stata quindi condotta un'analisi "intention-to-treat" (ITT) e ITT modificata (in cui il clinico conosceva il risultato). L'outcome principale era la prescrizione di antibiotici, mentre outcomes secondari erano prescrizione di antivirali, permanenza nel PS, ricovero ospedaliero, e controlli clinici ricorrenti. Di 931 visite al PS, un totale di 795 test RRP sono risultati positivi (85\%). Nel gruppo di intervento ITT, $i$ bambini hanno ricevuto più frequentemente antibiotici, senza differenze in prescrizione di antivirali, visite mediche successive, $e$ ospedalizzazione. Viceversa, nel gruppo ITT modificato, i bambini con esito del test noto hanno ricevuto più antivirali e sono stati più ospedalizzati, mentre non è risultata una differenza significativa nella prescrizione di antibiotici. In conclusione, lo studio dimostra che l'utilizzo di test RRP nel PS in bambini con sintomatologia ILI non ha ridotto la prescrizione di antibiotici, e che in generale esiste un ruolo limitato nell'utilizzo del test RRP in questo setting.
\end{abstract}

Rapid respiratory pathogen testing and antibiotic prescribing in ED in children with acute respiratory illness

There is high usage of antibiotics in the emergency department (ED) for children with acute respiratory illnesses. Studies have reported decreased antibiotic use among inpatients with rapid respiratory pathogen $(R R P)$ testing. The present study aims to determine whether ED RRP testing leads to decreased antibiotic use and health care use among children with influenza-like illness (ILI) in an ED. A randomized clinical trial among children aged 1 to 18 years presenting to an ED with ILI from December 2018 to November 2019 was conducted. Patients were randomized in an intervention group or a control group (results not given, routine clinical care). Intention-to-treat analyses and modified intention-to-treat (clinician knows results) analyses were conducted. Primary outcome was antibiotic prescribing whereas influenza antiviral prescribing, ED length of stay, hospital admission, and recurrent health care visits were the secondary outcomes. Among 931 ED visits, a total of $795 R R P$ test results (85\%) were positive. In the intention-to-treat intervention group, children were more likely to receive antibiotics, with no significant differences in antiviral prescribing, medical visits, and hospitalization. In inverse propensity-weighted modified intention-to-treat analyses, children with test results known were more likely to receive antivirals and be hospitalized; there was no significant difference in antibiotic prescribing. The use of RRP testing in the ED for ILI did not decrease antibiotic prescribing in this randomized clinical trial. There is a limited role for RRP pathogen testing in children in this setting.

\section{Background}

Le infezioni respiratorie acute simil influenzali (ILI) rappresentano una delle più comuni cause di accesso al Pronto Soccorso (PS) e di visita pediatrica urgente. Si stima che nel PS pediatrico $55-57 \%$ dei bambini ricevano una prescrizione di antibiotici, indipendentemente dal tipo di infezione, la maggior parte delle quali è virale. La prescrizione inappropriata di antibiotici può portare ad eventi avversi, aumento dei costi sanitari, resistenze agli antibiotici. L'identificazione del patogeno respiratorio costituisce un'opportunità per la corretta gestione del bambino con ILI, con potenziale riduzione dell'utilizzo di antibiotici, di utilizzo di risorse e costi sanitari. Le nuove piattaforme molecolari costituiscono un valido strumento per una rapida identificazione dei patogeni con alta sensibilità e specificità. Sebbene alcuni studi abbiano dimostrato che queste nuove tecnologie hanno portato ad una netta riduzione della prescrizione di antibiotici e dei tempi di ospedalizzazione, ci sono solo limitate evidenze che l'utilizzo di tali strumenti in PS pediatrico comporti gli stessi benefici.

\section{Scopi}

L'outcome primario di questo studio era di determinare se la conoscenza del patogeno responsabile di ILI potesse orientare la decisione del clinico riguardo la prescrizione di antibiotici e la gestione dei controlli clinici in un setting di PS pediatrico. Outcomes secondari includevano la prescrizione di farmaci antivirali antinfluenzali e la sua appropriatezza, la permanenza in PS, il ricovero ospedaliero, il numero di visite ricorrenti in PS, il numero di visite pediatriche entro 10 giorni dalla visita in PS, la decisione del clinico in PS (prescrizione di test e trattamenti), 
l'accettazione del test da parte dei pazienti/famiglie. L'ipotesi era una riduzione della prescrizione antibiotica nel gruppo di pediatri che fossero a conoscenza dell'esito del test rapido per patogeni respiratori (RRP).

\section{Metodi}

È stato approntato uno un trial clinico randomizzato utilizzando un campione di pazienti 1 mese - 18 anni che si presentava in PS del Children's Hospital Colorado con sintomi di ILI da dicembre 2018 a novembre 2019. ILI era stata definita come una sindrome influenzale con $\mathrm{TC}>37.8^{\circ} \mathrm{C}$, e almeno uno dei seguenti sintomi: tosse, faringodinia, congestione o scolo nasale, in bambini 12 mesi-18 anni; per i più piccoli ( $<12$ mesi) era sufficiente una TC $>37.8^{\circ} \mathrm{C}$ o uno dei suddetti sintomi. Tutti i bambini arruolati effettuavano un tampone nasofaringeo ed i risultati erano ottenuti in circa 45'; i patogeni esaminati erano: adenovirus; coronavirus HKU1, NL63, 229E, OC43; human metapneumovirus; rhinovirus/enterovirus; virus respiratorio sinciziale; virus influenza $\mathrm{A}$, A/H1-2009, A/H3, and B; virus parainfluenza 1, 2, 3, 4; bordetella pertussis e parapertussis; chlamydophila pneumoniae e mycoplasma pneumoniae. I risultati del test venivano forniti al clinico e alle famiglie del bambino nel gruppo di intervento, ma non nel gruppo di controllo. Il team coinvolto nello studio raccoglieva le caratteristiche sociodemografiche dei pazienti e i sintomi di presentazione dei bambini attraverso un report e il record medico, e provvedevano a raccogliere l'esame fisico, le diagnosi e l'outcome clinico attraverso l'analisi del record medico. I bambini venivano quindi randomizzati 1:1 nel gruppo di intervento (in cui il clinico conosceva l'esito del test) e in quello di controllo (risultato del test non fornito al clinico). L'analisi delle caratteristiche sociodemografiche e cliniche dei 2 gruppi è stata eseguita mediante il Pearson $\chi 2$ test e test non parametrici (Wilcoxon rank-sum test). Le analisi dei gruppi intention-to-treat (ITT) e ITT modificato (in cui il clinico conosce il risultato) sono state condotte utilizzando il sistema multivariabile di regressione di Poisson.

\section{Risultati}

Di 931 bambini visitati in PS (gruppo di intervento 452 bambini e gruppo di controllo 456 bambini, dopo l'esclusione di quelli che non soddisfacevano i criteri di inclusione), un totale di 795 test RRP sono stati eseguiti e risultati positivi nel $85 \%$ dei casi (Figura 1). L'età mediana dei bambini era di 2.1 anni, 509 (56\%) erano maschi. Nel gruppo di intervento ITT, i bambini avevano maggiori probabilità di ricevere antibiotici, senza differenze significative nella prescrizione antivirale, nelle visite mediche e nel ricovero in ospedale. Nell'analisi ITT modificata ponderata, i bambini con risultati dei test noti avevano maggiori probabilità di ricevere antivirali e di essere ricoverati in ospedale; non vi è stata alcuna differenza significativa nella prescrizione di antibiotici (Tabella). Le decisioni cliniche sono state modificate nel $17 \%$ dei casi (72 su 435) basandosi sui risultati dei test RRP. Quando sono stati osservati cambiamenti nel processo decisionale clinico, era più comunemente prescritto l'antivirale (prescrizione oseltamivir, $\mathrm{n}=11$; non prescrizione oseltamivir, $\mathrm{n}=15$ ) (Figura 2)

\section{Discussione}

Figura 1. Patogeni identificati nei bambini arruolati nello studio mediante test RRP

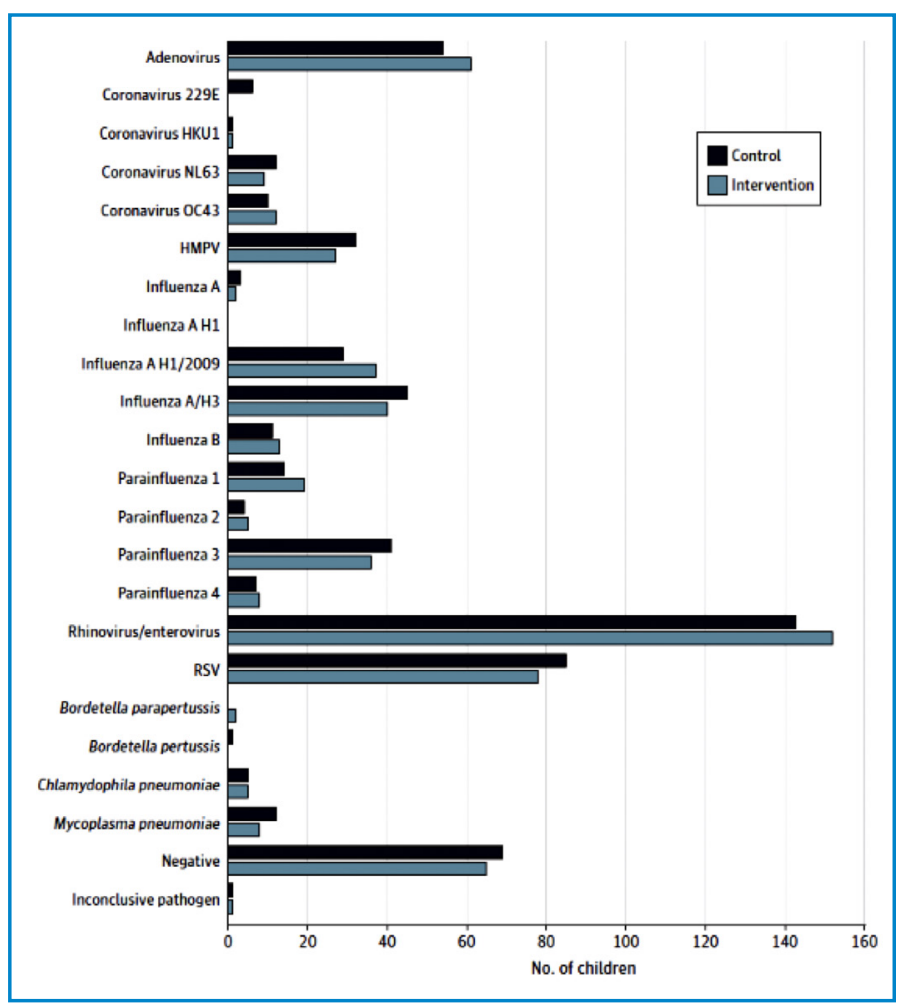

Figura 2. Processo decisionale clinico nel pronto soccorso in base ai risultati di test rapidi per agenti patogeni respiratori

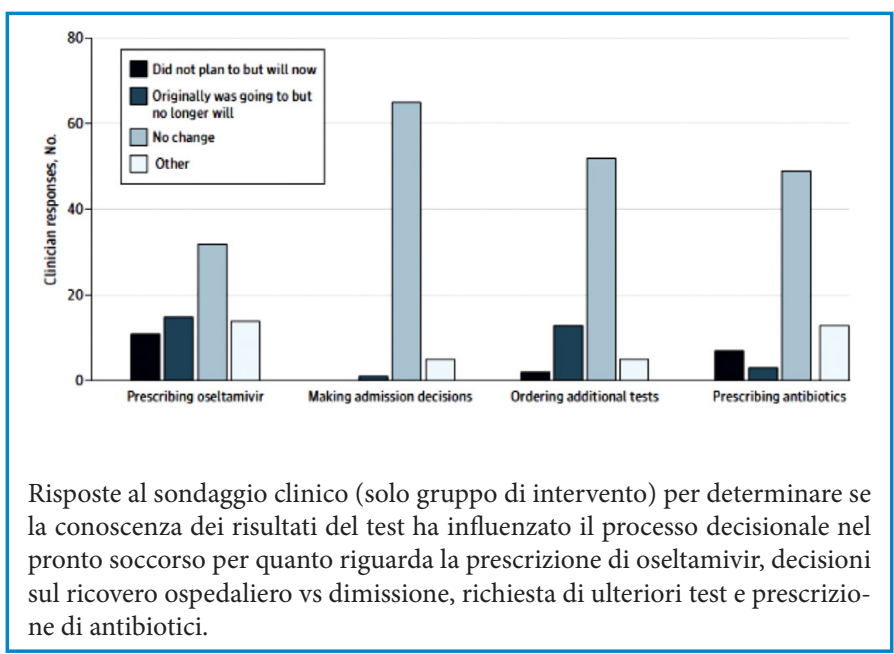

Lo studio (il più grande RCT sull'argomento) valuta l'utilità clinica del test rapido RRS in un ambiente pediatrico di emergenza. Alcuni risultati di metanalisi avevano evidenziato la necessità di un grande studio clinico randomizzato per valutare la diagnostica molecolare rapida in un ambito di terapia intensiva pediatrica, utilizzando la prescrizione di antibiotici come endpoint primario, dati i limitati risultati esistenti e il piccolo numero di partecipanti agli studi precedenti. È stato rilevato che i bambini nel gruppo di intervento i cui medici erano a conoscenza dei risultati del test RRP avevano maggiori probabilità di ricevere antibiotici, senza differenze significative nella prescrizione di antivirale, di durata della degenza, di successive visite in P.S. e di tassi di ospedalizza- 
Lo studio valuta l'utilità clinica del test rapido RRS in un ambiente pediatrico di emergenza, principalmente al fine di valutare la possibilità di riduzione della prescrizione di antibiotici utilizzando il suddetto test. I risultati degli autori rivelano che l'utilizzo del test RRP in PS pediatrico non determina una diminuzione della prescrizione di antibiotici, outcome primario dello studio. Gli autori evidenziano peraltro una maggiore appropriatezza nella prescrizione dell'antivirale allorché si utilizzi il pannello RRS, a seconda che il test abbia rilevato o meno la presenza del virus influenzale. Suggeriscono quindi di considerare l'utilizzo del singolo test molecolare rapido per il virus influenzale, piuttosto che dell'intero pannello per patogeni respiratori.

In conclusione, sembra chiaro che utilizzare test RRS per tutti i bambini con ILI che giungono in PS è uno spreco di risorse e tempo, che non modifica il comportamento del pediatra dell'emergenza. Forse anche il test per il virus influenzale non sembra particolarmente utile, almeno nella realtà nostra locale, in quanto la prescrizione di antivirali in età pediatrica in PS è limitata solo ad alcuni casi specifici e non è raccomandata di routine [4]. Personalmente, continuo a credere che l'esame clinico risulti ancora oggi la strada maestra anche nei casi di ILI, che indirizzi la richiesta di un test di laboratorio ed eventualmente la prescrizione di una terapia.

1. Doan Q, Enarson P, Kissoon N, Klassen TP, Johnson DW. Rapid viral diagnosis for acute febrile respiratory illness in children in the emergency department. Cochrane Database Syst Rev. 2014;(9):CD006452. doi:10.1002/14651858.CD006452.pub4

2. Doan QH, Kissoon N, Dobson S, et al. A randomized, controlled trial of the impact of early and rapid diagnosis of viral infections in children brought to an emergency department with febrile respiratory tract illnesses. J Pediatr. 2009;154(1):91-95. doi:10.1016/j.jpeds.2008.07.043

3. Messacar K, Hurst AL, Child J, et al. Clinical impact and provider acceptability of real-time antimicrobial stewardship decision support for rapid diagnostics in children with positive blood culture results. J Pediatric Infect Dis Soc. 2017;6(3):267-274.

4. Tagarro A, Cruz-Cañete M, Otheo E, et al.; en nombre del Grupo de trabajo de Infecciones Respiratorias de la Sociedad Española de Infectología Pediátrica (SEIP); Miembros del Grupo de Trabajo de Infecciones Respiratorias de la SEIP que han participado en la revisión del manuscrito. Oseltamivir para el tratamiento de la gripe en niños y adolescentes [Oseltamivir for the treatment of influenza in children and adolescents]. An Pediatr (Engl Ed). 2019 May;90(5):317.e1-317.e8. Spanish. doi: 10.1016/j.anpedi.2019.01.009. Epub 2019 Feb 21. PMID: 30797703. 